\title{
Long Term Implications of Covid-19 Around the Globe
}

\author{
Shikha Mishra and Swaroopa Chakole \\ ${ }^{1}$ Department of Community Medicine, Jawaharlal Nehru Medical College, Datta Meghe Institute of Medical \\ Sciences (Deemed to be University), Sawangi (Meghe), Wardha-442001, Maharashtra, India. \\ Corresponding author email: drswaroopachakole@gmail.com
}

\begin{abstract}
After first outbreak of coronavirus disease 2019 reported in Wuhan city of Hubei province of China, no one can claim that he or she has grasp on the pathophysiology of the disease. Still researchers and medical professionals are coming up with new trends and coronavirus still is unfolding another one's day by day. In the initial days of the pandemic the symptoms reported among infected patients were cough, cold, fever etc. with varying degree. As soon as researchers started to establish these symptoms as the only symptoms, some more and different symptoms were unfolded like diarrhea, dyspnea, loss of test and smell etc. therefore uncertainty attached to the coronavirus's behaviors is extremely high and also its geographical expanse is also vast. The treatment protocol also differs across continents. Antibiotics and vitamin supplements are the basic course which are given to patients with none to zero severe symptoms.Elderly, pregnant women and children are already in vulnerable category therefore they need extra attention to maintain their health condition. Long-term symptoms can be for long term and can severely damaging and affecting the normal lifestyle. Emotional and psychological implications are also quite overlooked as compared to other types of implications.Passing on intentionally or unintentionally, false information about exaggerated death figures, treatment methodologies, vaccine scare can really affect the containment measures negatively. People are checking information every now and then and there is no guarantee that the information they are seeing is authenticated or not. Future predictions based on pseudoscience; blind faith incidents are on rise creating a widespread social distress. This study intended to compile the varied influences of COVID-19 around the world.
\end{abstract}

\section{KEY WORDS: FATALITIES, DISTANCING, COVID-19, LONG TERM, IMPACT, TREATMENT, PSYCHOLOGICAL.}

\section{INTRODUCTION}

With more than a million case fatalities, COVID-19 becomes deadliest disease outbreak that has grappled the whole world. Since its inception from Wuhan city of the Hubei province of China, it has grown exponentially in terms of infection cases and deaths due to it. Due to its crown shapedphysiology, it is named as coronavirus. It is second in the family of coronaviruses and also called as SARS-COV-2 or novel coronavirus. As of December $19,2020,75,790,260$ infection cases have registered of

Biosc Biotech Res Comm P-ISSN: 0974-6455 E-ISSN: 2321-4007

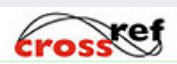

Identifiers and Pagination

Year: 2021 Vol: 14 No (6) Special Issue

Pages: 192-196

This is an open access article under Creative

Commons License Attribn 4.0 Intl (CC-BY).

DOI: $h t t p: / / d x . d o i . o r g / 10.21786 / b b r c / 14.6 .41$ which more than 42 million cases has be recovered(1-5). Unfortunately, this pandemic has taken a death toll of over a million and to be precise $1,677,594$ people lost their lives due to COVID-19 complications. Recovery rate is impressive over 95 percent and case fatality rate stands at little more than 3 percent (2).

But after converting that 3 percent it becomes a whooping million and half and which is also due to the pan global expanse of the pandemic. The pandemic containment strategy also contains the treatment procedure and often seen as the last step towards getting rid of the pandemic. But after one year into pandemic cases with persistence of symptoms after getting fully treated and discharged of COVID-19 started emerging. Widespread reporting of headache, severe fatigue, loss of taste and smell and many more to severecardiovascular, pulmonary and psychological impairment that may persists for 12 to 24 months post illness(3). Various sections of society have been affected in various ways.

\section{Article Information \\ Received: $29^{\text {th }}$ March 2021} ccepted after revision: $29^{\text {th }}$ May 2021 
For example, athletes need constant physical exercise to keep themselves fit. They are not getting enough opportunities to access the gymnasium or the health club which can severely hamper their physical as well as social wellbeing. Elderly, pregnant women and children are already in vulnerable category therefore they need extra attention to maintain their health condition. Longterm symptoms can be for long term and can severely damaging and affecting the normal lifestyle. Various preventive measures has been in practice to ensure the protection from the viral infection(4).

A Case for Long Covid-19: The sheer number of infected people worldwide gives idea about how rapidly it can progress. And the case fatality rate also a major cause of concern shows its deadliness. Even if a year has been passes recently after first outbreak of coronavirus disease 2019 reported in Wuhan city of Hubei province of china, no one can claim that he or she has grasp on the pathophysiology of the disease. Still researchers and medical professionals are coming up with new trends and coronavirus still is unfolding another one's day by day. In the initial days of the pandemic the symptoms reported among infected patients were cough, cold, fever etc. with varying degree. As soon as researchers started to establish these symptoms as the only symptoms, some more and different symptoms were unfolded like diarrhea, dyspnea, loss of test and smell etc. therefore uncertainty attached to the coronavirus's behaviors is extremely high and also its geographical expanse is also vast.

The treatment protocol also differs across continents. Antibiotics and vitamin supplements are the basic course which are given to patients with none to zero severe symptoms. Then there is medium severe category of patients suffering from shortness of breath and needs extra attention in the form of oxygen support system. Critical patients experiencing multiple organ failure needs Intensive critical care unit attention as they may need surgery for ailments. Whole world is affected by the pandemic so the outcomes are bound to be different. This also highlighted the needs of continuous and comprehensive analysis of the data that is arising out of multiple data points( Ngai et al, 2010). Also, as the along with current infection numbers there are many more people who got related and discharged from the disease. But the problem is that some of them are experiencing persisted and new symptoms even after getting negative for infection reports.

Long Term Implications of Sars and Mers: While studying the various aspects of COVID-19, due to its newness, it is difficult to draw trajectory of the diseases various impacts. Therefore, it becomes necessary to study the previous similar outbreaks like severe acute respiratory syndrome (SARS) andMiddle Eastern respiratory syndrome (MERS). A lot can be taken from the outbreak's management as a lesson and same can be useful in tackling the COVID19. In SARS outbreak intensive study after the outbreak was controlled was done and it has inferences that can at least extrapolated to predict the future outcomes in terms of the disease's long-term implications.
It is found out that different age groups, which turn out to the long-term assessment post SARS infection of over twelve to twenty-four months, have different impacts on their physical health, exercise capacity and especially pulmonary function which is primly affected by the infection. Younger age groups were recovering quickly barring few comorbid individuals which too shown some positive signs of speedy recovery. Whereas comorbid and relatively aged population was struggling to keep up the pace of recovery with their younger counterparts.

Study found out that overall 52 percent patients of SARS post illness were found to be affected with impairment in diffusing capacity of the lungs for carbon monoxide (DLCO)and reduced capacity of exercise and health status after 24 months after they got discharged from the SARS treatment. 78 percent of the SARS affected people returned to their pre-SARS era jobs and livelihoods and nearly 30 percent health care professionals were not able to resume their duty. Although DLcolevels were found to be improving after a year in some patients outside the study still it established that pulmonary functions can be severely impaired even if one does get cured of respiratory syndrome. Weakness in respiratory muscular function and decreased lung capacity was among prime negative impacts that patients had to face post illness. The impairment in DLco value was further found to be affecting the exercising ability and physical health status.

This impairment generally found to be persisting for 14 months post illness. In addition, few patients were found to be affected by post-trauma disorder, anxiety and mild levels of depression. Prolonged bed rest can be one contributing factor along with isolation and distancing from the friends and family members. The less number of health care professionals were reported to rejoin can be attribute to the excessive hostile environment and constant physical and mental stress induced by SARS which takes longer to stable the conditions(6). In case of 2012 outbreak of MERS somewhat similar outcomes were observed among patients followed up to two years post illness and discharge. The pulmonary function and physical capacity were found to negatively affected and concerned people were found to be struggling with dayto-day activities.

In HongKong a 36-item short form survey (SF-36) scores were found to be way less than those in unaffected population. Although the survey did not take in to account the mental as well as emotional implications. Those patients who were administered corticosteroids while their medical stay in Intensive care unit were found to be suffering from muscle wasting and reduced quality of life. Critical illness in patients induced post trauma disorder which was also seen in SARS during follow up. Prolong stay in hospital and isolated condition may also have role in inducing depression among them. It is observed that patients in both SARS and MERS outbreaks which are admitted to ICU for critical care were lagging by considerable margin from their ward 
counterparts in terms of psychological, physical and social wellbeing(7).

Long Term Implications of Covid-19: As the COVID-19 is progressing lots of patients are also being recovered from the disease, but previous studies done after similar outbreaks of SARS and MERS shows that the story is not over yet. Patients' needs to be followed up for considerable amount of time in order to assess the situation comprehensively. It is one year after pandemic has struck but the persistence of the symptoms and reduced physical capacity complaints has started to arise six months post onset of the pandemic. Various conditions like cough, fatigue, periodic shortness of breath, pain in the chest and joints of the body, difficulty in concentrating and in processing thoughts also known as brain fogging, muscle pain, palpitations, intermittent loss of taste and smell and many more.(Rastogi et al., 2020) Symptoms are varied and needs proper study to deal with it.

The reason behind these post illnessesmedical condition is not yet known. Persistence of these condition may last from few weeks to few months but not exactly predictable as pandemic of COVID-19 is still evolving. Moreover, in some cases Cardiovascular, respiratory, neurological as well as psychiatric conditions were started to arise post illness. Which is a serious cause of concern because mere containment is not the option to deal with the COVID-19.Cardiovascular implications include myocardial injury especially after increased level of troponin while in treatment of COVID-19. As the gateway of the virus is the respiratory system but it does not limited to it(Rimmer, 2020). Major organ system of the human anatomy is affected one way or the other. While in severe illness occurring due to COVID-19, the cytokine storm which is intense may affects the cardiac function in negative way.

But it is confined to critically ill patient of COVID-19. In another scenario COVID-19 can affect myocardial cells and can infect them and can lead to myocarditis in long term post illness. The vulnerability to ventricular premature complexes like malignant ventricular arrhythmias increases if the intensity of the cytokine storm is too high. People doing athletics have chances of developing the myocardial inflammation that lingers long after the illness has been treated. Radiological imaging shown that there was a long-lasting impact on the heartmuscle even after mild symptoms were exhibited by the patient. This can increase the chances of severe heart ailments in the future. Neurological implications are profoundly seen as brain fog which state of confusion and difficulty in decision is making ability, vertigo which was not present earlier, anosmia, seizures and many more.

This has been reported in COVID-19 patients after three to four months post illness (Yan et al, 2020). In SARS and MERS too, there were similar neurological implicationsleading to encephalitis etc. Cognitive health and day to day functioning ability is marred by the unidentified post infection illness. More study needed in order to establish certain impairment as post COVID-19 illness. Observing the previous outbreak's data, more likely long term implications are only possible scenario that will persists(4). Emotional and psychological implications are also quite overlooked as compared to other types of implications. Often neglected, can have extremely profound impact on the overall wellbeing of the person post infection from the COVID-19.

Mild to critically ill patients were found to be more affected by this as they were isolated and quarantined for considerable amount of time. Feeling of missing out on major events, not able to meet friends and family members, physical distancing measures were found to be inducing anxiety and depression not only in infected patients but also in general populace as their loved ones were also affected by the grim scenario that the world is facing now. Substance abuse and withdrawal symptoms were on peak as the consumption of sin goods were unavailable suddenly during lockdown period. Athletes and sports persons are finding it difficult to cope up with regular exercise quota to get them fit. This can induce uncertainty and depressionas the future is quite unpredictable and the carrier trajectory seems blurred. Elderly and pregnant women are on the verge of falling for psychosocial distress as their medical condition is already in a weakened state(Yan et al, 2020).

Children's and even pet animals are in distress as they are not able to comprehend properly what sudden and drastic changes would mean for them. They cannot roam and play outside affecting their growth and they need physical activities the most. They need extra attention in terms of being mentally well. Pregnant women are constantly anxious about transmitting the infection to their offspring. Although vertical transmission is not yet established but physical transmission post-delivery may happen due to human negligence while handling the baby. Infodemic is yet another aspects that only worsens the situation( Gualano et al., 2020). Passing on intentionally or unintentionally, false information about exaggerated death figures, treatment methodologies, vaccine scare can really affect the containment measures negatively.

People are checking information every now and then and there is no guarantee that the information they are seeing is authenticated or not. Future predictions based on pseudo-science; blind faith incidents are on rise creating a widespread social distress. The uncertainty attached to the COVID-19 is huge and nobody has concrete answer about the future (Lancet, 2020). As the vaccine is entering in mass production and distribution phase after obtaining the required permissions, various type of vaccine scare and hesitancy are peeking out on baseless facts. This will create a huge roadblock in vaccination drive of the population. It may create another disaster parallel to COVID-19. 
Impact on Athletes: A special case arises with respect to athletes as the pandemic is progressing. Al ready due to various measures of lockdown and physical distancing almost all sports were hibernating and players were confined to their residence. No practice sessions and physical fitness camps were running and this hinders in maintaining the physique of the player. Regular exercise and endurance training are the corner stone of any sport. Then the case worsens if player becomes positive for infection of COVID-19(14). The treatment can be easily done as players are fit and free of any major comorbidity but the long-term post COVID-19 impactmay be profound and can hinder with their previous capacity.

Particularly the DLCO levels impairment and cardiovascular muscle weakening can be observed in some cases. This can take almost couple of years to completely recover. Pre participation evaluation needs to done before admitting any player into any physical fitness camp. Millions of player'scarriers at stake and more comprehensive research are needed to draw out any rehabilitation plan if necessary. Regular testing is necessary in order to detect the infection as soon as possible to avoid complications that happens in later stage of the infection (Baggish et al., 2020).

Preventive Measures: Since so far, we have seen some critical after effects of the COVID-19 which can last long, enough to be detrimental to the functioning of the body. So, it is advisable to not getting contracted with the virus at first place to ensure that there are no post illness implications which seems to be more damaging the illness itself. Various guidelines are issued by governing authorities to ward off the virus. Wearing masks, gloves, personal protective equipment kit, face shields with following physical distancing norms and roaming outside only when necessary is the basic mantra for not getting infected. We can resume our life with these precaution(16). Related studies reported from this region were reviewed(Ather et al, 2020; Shah et al., 2020). Implications of Covid -19 reflected in the reviews of (Butola et.al., 2020; Burhani et. al., 2020; Arora et. al., 2020; Dhankasar et. al., 2020; Patel et.al.,2020).

This will not only help us in keeping ourselves safe but also will help us in preventing other communicable disease's infection. Maintaining hygiene is essential not only till the pandemic persists but all the time as hygienic conditions keeps various diseases at bay. Health care professionals need to be extra cautious in order to remain infection free as already the health care personnel to per unit population is quite low in about all the countries, least developed countries being worse affected. Also, vaccine as in final stage is not meant for all at once. The distribution of dosage to be in phase wise manner. Some of them might not even get one until few months from now so it is important to take all precaution and follow best practices available to avoid infection. This will not only protect you but also will aid in the global fight against the pandemic.

\section{CONCLUSION}

The pandemic is not going anywhere until for minimum of one year from now. Even after vaccine arrives, which is still in final phases, we have to live with the virus. Vaccine is not the panacea and more needs to be done. The long-term health impact is too serious and needs to study in depth. We can prepare model to tell people that preventing is way better than cure when the long-term lifestyle is concern. Especially cardiovascular impact is profound and can prove critical in future. By inculcating various measures like wearing masks, physical distancing we can ensure that we can break the chain of the viral transmission. Athletes and sportspersons can be given limited permission to practice and for physical exercise in stadiums with proper precaution. We have to live with this pandemic and changing our lifestyle in positive way will make that thing easier. Still no official study to follow up the patients after treatment of COVID-19 has been sanctioned. There is a dire need to address this concern and patients should be followed up closely.

Post COVID-19 rehabilitation centers needs to be established in order to accommodate huge number of people that will make similar complaints in future. Lessons from SARS and MERS have lot to offer and we should tale cue from them to be ready for the post infection persistence of symptoms scenario. Allied health care professionals can be trained to treat the long COVID19 patients to reduce the burden on doctors treating the COVID-19 patients. Finally, awareness needs to be created in order to medically literate the people about the virus and its consequences. Professing people, the importance of hygiene and telling them about leaving bad habits if any can help not only now but in future too. As WHO said that COVID-19 is not the last pandemic that world has and is being seen but there can be one in future may be similar or may be more severe than this. We should be ready to deal with any situation with positively changing our lifestyle and inculcating good habits.

\section{REFERENCE}

Arora, Devamsh, Muskan Sharma, Sourya Acharya, Samarth Shukla, and Neema Acharya. 2020 India in 'Flattening the Curve' of COVID-19 Pandemic - Triumphs and Challenges Thereof." JOURNAL OF EVOLUTION OF MEDICAL AND DENTAL SCIENCES-JEMDS 9, no. 43 (October 26, 2020): 3252-55.

Ather B, Mirza TM, Edemekong PF. 2020 Airborne Precautions. StatPearls [Internet], StatPearls : https:// www.ncbi.nlm.nih.gov/books/NBK531468/

Baggish A, Drezner JA, Kim J, Martinez M, Prutkin JM. 2020 Resurgence of sport in the wake of COVID19: cardiac considerations in competitive athletes. Br J Sports Med https://www.ncbi.nlm.nih.gov/pmc/articles/ PMC7513253/

Batawi S, Tarazan N, Al-Raddadi R, Al Qasim E, Sindi A, AL Johni S, et al. 2020 Quality of life reported by survivors after hospitalization for Middle East respiratory syndrome (MERS). https://doi.org/10.1186/ 
s12955-019-1165-2

Burhani, Tasneem Sajjad, and Waqar M. Naqvi. 2020 Telehealth - A Boon in the Time of COVID 19 Outbreak." JOURNAL OF EVOLUTION OF MEDICAL AND DENTAL SCIENCES-JEMDS 9, no. 29 (July 20: 2081-84.

Butola, Lata Kanyal, Ranjit Ambad, Prakash KeshaoraoKute, Roshan Kumar Jha, and Amol Dattaroa Shinde. "The Pandemic of 21st Century - COVID-19." JOURNAL OF EVOLUTION OF MEDICAL AND DENTAL SCIENCES-JEMDS 9, no. 39 (September 28, 2020): 2913-18. https://doi.org/10.14260/jemds/2020/637.

CDC. COVID-19 and Your Health [Internet]. Centers for Disease Control and Prevention. 2020 [cited 2020 Dec 17]. Available from: https://www.cdc.gov/ coronavirus/2019-ncov/long-term-effects.html COVID-19 (coronavirus): Long-term effects [Internet]. Mayo Clinic. [cited 2020 Dec 17]. Available from: https://www.mayoclinic.org/diseases-conditions/ coronavirus/in-depth/coronavirus-long-term-effects/ art-20490351

COVID-19 cardiac injury: Implications for long-term surveillance and outcomes in survivors https://www. ncbi.nlm.nih.gov/pmc/articles/PMC7319645/

COVID-19 Map [Internet]. Johns Hopkins Coronavirus Resource Center. [cited 2020 Dec 19]. Available from: https://coronavirus.jhu.edu/map.html

Dhankasar, Priya, Pallavi Dhole, Seema Kolhe, MinalDambare, Priya Rewatkar, and Vaishali Balpande. "The Unseen Positive Effect of Covid-19 Pandemic." JOURNAL OF RESEARCH IN MEDICAL AND DENTAL SCIENCE 8, no. 6 (2020): 108-12.

Gaidhane S, Khatib N, Zahiruddin QS, Gaidhane A, Telrandhe S, Godhiwal P. 2020 Depression, anxiety and stress among the general population in the time of COVID-19 lockdown: A cross-sectional study protocol.' International Journal of Research in Pharmaceutical Sciences, 11(1) pp. 360-364.

Gualano MR, Lo Moro G, Voglino G, Bert F, Siliquini R. 2020 Effects of Covid-19 Lockdown on Mental Health and Sleep Disturbances in Italy. Int J Environ Res Public Health [Internet]. https://www.mdpi.com/16604601/17/13/4779

Jachak, S., P. Phansopkar, and M. Waqar Naqvi. 2020 Impact of Covid-19 in India, a Disastrous Pandemic Outbreak." International Journal of Research in Pharmaceutical Sciences 11, no. Special Issue 1: 399402. https://doi.org/10.26452/ijrps.v11iSPL1.2735.

Joseph, M.B., S. Pohekar, A. Raut, and M. Patil. 2020 The Palliative Care and Covid-19 Pandemic.” International Journal of Research in Pharmaceutical Sciences 11: 618-22. https://doi.org/10.26452/ijrps.v11iSPL1.2861. Joshi, K., N. Acharya, S. Acharya, and S. Joshi. 2020 A Grave Situation with COVID in the Gravid: A Narrative Review." International Journal of Research in Pharmaceutical Sciences 11: 496-99. https://doi. org/10.26452/ijrps.v11iSPL1.2837.
Khatib, M.N., S. Gaidhane, M. Khatib, M. Ahmed, A. Gaidhane, and Z.Q. Syed. 2020 SARS-CoV and SARSCoV-2: Similar Viruses with Different Trajectories." Wutan Huatan Jisuan Jishu 16(5): 544-48.

Lakhkar, B.B., B. Guru, S. Damke, and S. Damke. 2020 Most Susceptible Duo in COVID-19 Crisis: A Literature Review." Perinatology 21: 112-23.

Latchoumi, T.P., Ezhilarasi, T.P. and Balamurugan, K., 2019. Bio-inspired weighed quantum particle swarm optimization and smooth support vector machine ensembles for identification of abnormalities in medical data. SN Applied Sciences, 1(10), pp.1-10.

Ngai JC, Ko FW, Ng SS, To K-W, Tong M, Hui DS. 2010 The long-term impact of severe acute respiratory syndrome on pulmonary function, exercise capacity and health status. Respirol Carlton Vic., 15(3):543-50. Patel, Mohan P., Vivek B. Kute, Jitendra Goswami, and Manish R. Balwani. 2020 Hospitals May Become 'Disease Hotspots' for COVID-19 Amid Shortage of Personal Protective Equipment." INDIAN JOURNAL OF CRITICAL CARE MEDICINE 24: 1145-46.

Rastogi A, Tewari P. 2020 Covid 19 and its cardiovascular effects. Ann Card Anaesth, 23(4):401.

Rimmer A. 2020 Covid-19: Impact of long term symptoms will be profound, warns BMA https://www. bmj.com/content/370/bmj.m3218

Shah, P., and Naqvi W. 2020 Fighting And Chasing The Rogue Virus-Covid19'. International Journal of Research in Pharmaceutical Sciences, 11(1), pp. 77-80.

The Lancet null. India under COVID-19 lockdown. Lancet Lond Engl. 2020 25;395(10233):1315.

The long-term health effects of COVID-19 [Internet]. [cited 2020 Dec 17]. Available from: https://www.gavi. org/vaccineswork/long-term-health-effects-covid-19 Toshida, T., and Chaple J. 2020 Covid-19 - Rumours and Facts in Media'. International Journal of Research in Pharmaceutical Sciences, 11(1), pp.171-74. pharmascope. org, doi:10.26452/ijrps.v11iSPL1.2344.

What we know about Long-term effects of COVID-19 : https://www.who.int/docs/default-source/coronaviruse/ risk-comms-updates/update-36-long-term-symptoms. pdf?sfvrsn=5d3789a6_2 [Internet]. [cited 2020 Dec 17]. Available from: https://www.who.int/docs/defaultsource/coronaviruse/risk-comms-updates/update-36long-term-symptoms.pdf?sfvrsn=5d3789a6_2 WHO Coronavirus Disease (COVID-19) Dashboard [Internet]. [cited 2020 Dec 19]. Available from: https:// covid19.who.int

Yan J, Guo J, Fan C, Juan J, Yu X, Li J, et al. Coronavirus disease 2019 in pregnant women: a report based on 116 cases. Am J Obstet Gynecol, https://www.ajog.org/ article/S0002-9378(20)30462-2/abstract

Zhang L, Tao Y, Shen M, Fairley CK, Guo Y. Can selfimposed prevention measures mitigate the COVID-19 epidemic? https://journals.plos.org/plosmedicine/ article?id=10.1371/journal.pmed. 1003240 